Volume 8, No.5, September - October 2019

International Journal of Advanced Trends in Computer Science and Engineering

Available Online at http://www.warse.org/IJATCSE/static/pdf/file/ijatcse47852019.pdf

https://doi.org/10.30534/ijatcse/2019/47852019

\title{
Evaluation of the Potential Accuracy of Correlation Extreme Navigation Systems of Low-Altitude Mobile Robots
}

\author{
Alexander Tantsiura ${ }^{1}$, Dmytro Kolomiets ${ }^{2}$, Iryna Tabakova ${ }^{3}$, Iryna Hannoshyna ${ }^{4}$, Nataliia Serdiuk ${ }^{5}$, \\ Oleksandr Yelieazarov ${ }^{6}$, Tetiana Voichenko ${ }^{7}$ \\ ${ }^{1} \mathrm{PhD}$, Researcher, Department of Air Force Research Center, Kharkiv National University named after Ivan \\ Kozhedub Air Force, Ukraine, shurik.lucky@gmail.com \\ ${ }^{2}$ Senior Lecturer, Department of Navigation and Ship's Handling, State University of Infrastructure and \\ Technologies, Ukraine \\ ${ }^{3} \mathrm{PhD}$, Senior Lecturer, Department of Media Systems and Technologies, Kharkiv National University of Radio \\ Electronics, Ukraine, iryna.tabakova@nure.ua \\ ${ }^{4}$ Senior Lecturer, Department of Navigation and Ship's Handling, State University of Infrastructure and \\ Technologies, Ukraine \\ ${ }^{5} \mathrm{PhD}$, Associate Professor, Department of Computer Intelligence Technologies And Systems, Kharkiv National \\ University of Radio Electronics, Ukraine \\ ${ }^{6} \mathrm{PhD}$, Associate Professor, Department of Navigation and Ship's Handling, Dean of the Faculty of Navigation, \\ State University of Infrastructure and Technologies, Ukraine \\ ${ }^{7} \mathrm{PhD}$, Associate Professor, Department of Means of Transport Service (Inland Navigation),State University of \\ Infrastructure and Technologies, Ukraine
}

\begin{abstract}
The sight surface (SS) results for the generalized model image development of the correlation-extreme navigation systems (KENS) of mobile robots (MR) in the informative parameter step difference form used in the system are presented. The generalized telegraph process model usage possibility of the radiation emitter image in a wide MR spectral range is substantiated. To assess the KENS accuracy potential for this model type image the relations are obtained. The potential KENS positioning accuracy assessment for typical model conditions of MR usage is carried out. The estimates are made for the informative parameter used in radiometric navigation systems, which can be similarly obtained for other types of informative parameters.
\end{abstract}

Key words: mobile robot, extreme correlation navigation system, generalized telegraph process, potential accuracy.

\section{INTRODUCTION}

The mobile robots accuracy location, as one of the most important quality indicators, largely depends on the images describing method formed by one or another correlation-extreme navigation system, regardless of the informative parameter type used [1].

For the low-altitude MRs, most frequently used images are of the object against the background type, which are the surface sight images in the form of a step difference informative parameter along the flight direction. To determine the potential accuracy of the radiometric KENS MR the relations are obtained. Numerical evaluations of the MR potential accuracy navigation using a radiometer as an informative sensor and an image of a generalized telegraph process form are performed.

\subsection{Problem analysis}

The SS describing method when assessing the KENS quality functioning, regardless of the informative parameter type, can have a significant impact on the main characteristics of the navigation system, and, first of all, on the accuracy characteristics. There are various ways of presenting images, the choice of which is determined by both the features of the KENS construction and the conditions of their sight. For lowaltitude MRs, the most acceptable is the SS representation image along the flight line in the form of a step difference informative parameter described by the generalized telegraph process and taking into account the SS correlation properties [2]. The need for high MR navigation accuracy determines the appropriate studies conduct aimed at assessing the impact of the image presentation method on the KENS functioning quality.

The article aim is to assess the MR potential navigation accuracy equipped with KENS when using the informative parameter step difference along the flight line as images, which takes into account the SS correlation properties.

\section{MAIN MATERIAL}

2.1 The generalized sight surface model image formed by KENS

Various approaches are used to represent SS images. The analysis results of the known SS types images representation in different spectral ranges show that to the greatest extent of the photographing process the zone representation is adequate [3, 4]. The image band structure is formed due to the differences in the 
electrophysical properties of the SS cover sections, which in turn lead to differences in the reflective, radiating characteristics of the SS objects and, ultimately, their brightness characteristics. The zone SS description image model adequately characterizes the image scene and can be described by the mutual correlation function (MCF) of the generalized telegraph process and fully takes possible variations in the brightness of materials and covers into account, as well as the SS zone structure changing possibility. According to [5], the SS images have a non-Gaussian distribution law and contain constant in time zones with electrophysical characteristics of various covers and materials. The most characteristic stable features of the image, as a rule, are the boundaries of these SS unchanged sections (Figure 1) [6].
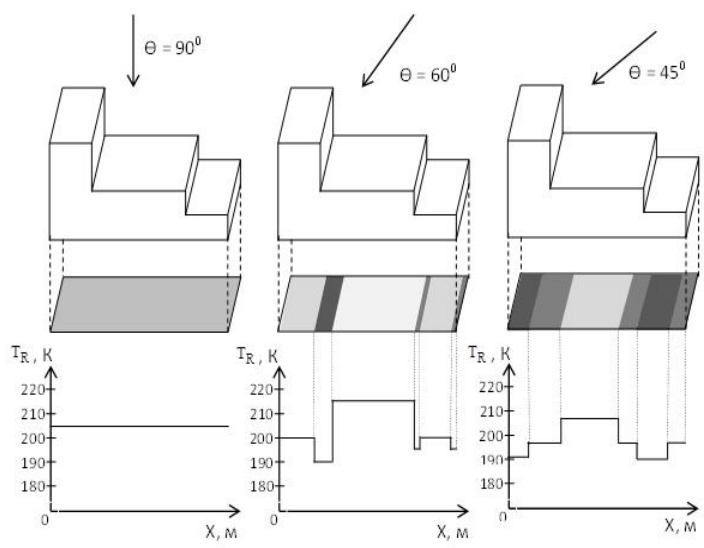

Figure 1 :Rradio brightness temperature distribution over the sight area: a) angle of sight $90^{\circ}$; b) sight angle $60^{\circ}$; c) viewing angle $45^{\circ}$

Therefore, the largest amount of the SS information in the image frame is concentrated on the zones boundaries $[2,3,6]$. At the same time, based on the random nature of the images filling that are being formed, the approach to their presentation should be statistical, and the analysis method should be correlation-spectral. The image similarity degree along with the deterministic reference image (template) is determined by calculating the MCF with the search for the extreme of this function, which determines the stochastic connection degree of the images. For deterministic images, there are optimal similarity measures. In the case under consideration, for the template and ensemble of images, the optimal measure of similarity does not exist. Therefore, given the fact that, under certain assumptions, KENS can be considered as linear systems, it is advisable to apply linear similarity measures, despite their simplicity and optimal for a Gaussian signal. It is known that any linear similarity measure can be expressed through a correlation function. Therefore, MCF [6] can be selected as the current image model (CI).

Since the probability density of the image is unknown, to determine the MCF image, we represent the sight surface $S_{C I}(t, \theta)$ as a set of homogeneous zones $S_{i}$ and integuments $A_{i}$, each of which is characterized by its brightness value $\mathrm{T}_{\mathrm{Ri}}$ which is the same within the k-th zone:

$$
\mathrm{S}_{\mathrm{CI \kappa}}(\mathrm{t}, \theta)=\mathrm{S}_{\mathrm{i}} \mathrm{T}_{\mathrm{Ri}} \mathrm{r}_{\mathrm{i}}(\mathrm{x}, \mathrm{y}, \mathrm{t}) \text {, }
$$

where $r_{i}(x, y, t)-$ is the indicator function $r_{i}(x, y, t)=1$, if $x, y \in A_{i}, r_{i}(x, y, t)=0$, if $x, y \notin A_{i}$.

Then, the SS image in representation (1) in any possible direction will look similar to the generalized telegraph process.

It is known [4] that the correlation function of such a process is described by the expression:

$$
\mathrm{R}(\mathrm{r})=\exp (-\alpha / \mathrm{r})
$$

where $\alpha=1 / \tau_{\mathrm{k}}-$ is the reciprocal of the correlation interval $\tau_{\mathrm{k}}$.

If we assume that the statistical properties of homogeneous surface zones are the same, then they can be considered isotropic. In this case, it is enough to determine the correlation function (2) by one coordinate ( $\mathrm{x}$ or $\mathrm{y}$ ).

In the general case, the image correlation function for an infinitely large sample, depending on the direction and size of the homogeneous zones, will be determined by the expression:

$$
\mathrm{R}(\overrightarrow{\mathrm{r}})=\exp (-\alpha / \overrightarrow{\mathrm{r}})
$$

For a finite large sample, the correlation function (3) will asymptotically approach the exponent.

Thus, a generalized SS image model in a wide spectral range can be represented by the correlation function of the generalized telegraph process for any SS part with the correlation interval corresponding to a specific region.

According to (3), the final expression for the decisive function (DF), taking into account the SS correlation properties, can be represented as follows:

$$
\mathrm{R}_{\mathrm{i}}\left(\mathrm{t}, \mathrm{r}, \theta, \theta_{\mathrm{i}}\right)=\mathrm{F}_{\mathrm{SP}}\left[\mathrm{F}_{\mathrm{RS}}(\exp (-\alpha / \mathrm{r}), \mathrm{n}(\mathrm{t})), \mathrm{S}_{\mathrm{RI}}\left(\theta_{\mathrm{i}}\right)\right],
$$

where $\mathrm{F}_{\mathrm{SP}}-$ is the comparison operator of the current $\mathrm{F}_{\mathrm{RS}}(\exp (-\alpha / \mathrm{r}))$ and reference $\mathrm{S}_{\mathrm{RI}}\left(\theta_{\mathrm{i}}\right)$ images;

$\mathrm{F}_{\mathrm{RS}}$ - an operator describing the CI forming process; $\mathrm{n}(\mathrm{t})$ - additive noise in the KENS channels.

Optical images can also be described by a generalized telegraph process, since the basis of image formation is the results of aerial photography, which determine their zone structure. 
Thus, a statistical approach to the description of the SS images has been substantiated, which allows the generalized telegraph process, representing the SSs with the corresponding correlation function, to be the basis of the image description model.

When filling the image scene obtained as a result of aerial photography of the area with brightness values, an image with a degree of detail, which is determined by the resolution of the photo image, can be obtained.

\subsection{Determination of the potential KENS radiometric accuracy}

Positioning in order to assess the potential accuracy of determining the radiometric KENS in accordance with (1), we will present the SS image in the form of a step wise difference in radio brightness temperatures along the line $x=a_{1}$. The geometric conditions for the formation of KENS CI MR are presented in Figure 2 [7].

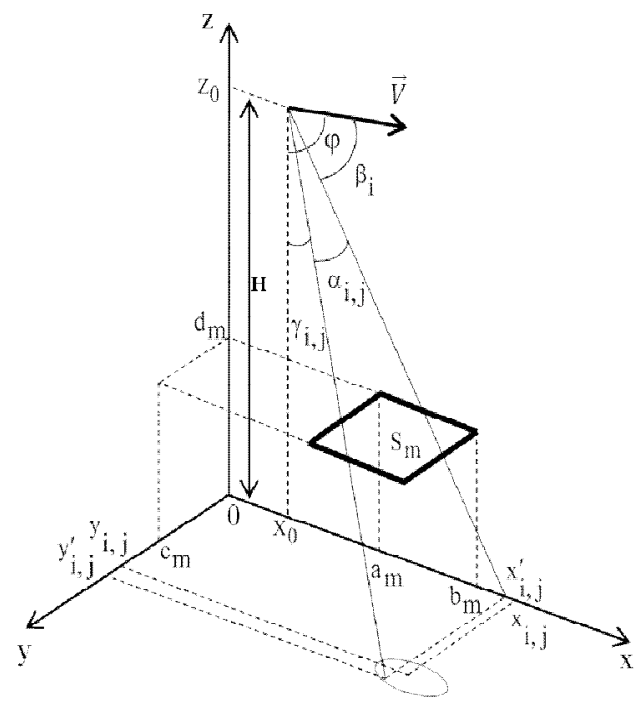

a)

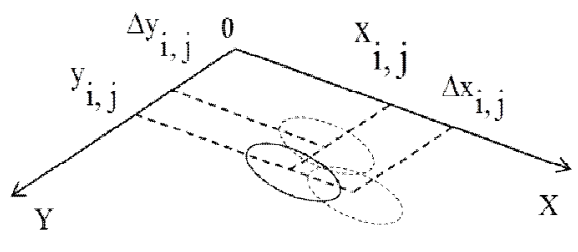

b)

Figure 2 : Geometric conditions for the formation of KENS CI MR: a) the antenna pattern position (APP) of the radiometric sensor in the xyz plane; b) partial APP when changing the spatial position of MR

$$
\mathrm{T}_{\mathrm{r}}(\mathrm{x}, \mathrm{y})=\left\{\begin{array}{c}
\mathrm{T}_{1},(\mathrm{x}, \mathrm{y}) \in \mathrm{S}_{1} ; \\
\mathrm{T}_{\phi},(\mathrm{x}, \mathrm{y}) \notin \mathrm{S}_{1},
\end{array}\right.
$$

where half-space $S_{1}$ is determined by the expression $S_{1}=\left\{(x, y) \in \mathbf{R}^{2}\left(x \geq a_{1}\right\}\right.$.

Thus, when using (5), it should be assumed $b_{1}=\infty$, $c_{1}=-\infty, d_{1}=\infty$. To simplify the problem, we also make the assumption that the channels characteristics of each row are the same, i.e. $\sigma_{\mathrm{i} 1}=\mathrm{L}=\sigma_{\mathrm{iN}}=\Sigma_{\mathrm{i}}$. In addition, we assume that the event that the difference line falls $\mathrm{x}=\mathrm{a}_{1}$ into the system's field of view is reliable. Then the output signals set of the multi-channel radiometric KENS can be written in accordance with (5) as follows:

$$
\begin{aligned}
& \tilde{\mathrm{h}}_{\mathrm{ij}}(\mathrm{t})=\mathrm{T}_{\phi}+\Delta \mathrm{T} \tilde{\mathrm{f}}_{\mathrm{i}}(\mathrm{t}, \varepsilon)+\mathrm{n}_{\mathrm{ij}}(\mathrm{t}), \mathrm{i} \in \overline{1, \mathrm{~N}_{1}}, \mathrm{j} \in \overline{1, \mathrm{~N}_{2}}, \\
& \tilde{\mathrm{f}}_{\mathrm{i}}(\mathrm{t}, \varepsilon)=1-\Phi\left(\frac{\varepsilon-\zeta_{\mathrm{i}}(\mathrm{t})}{\delta_{\mathrm{i}}}\right)+\exp \left[\begin{array}{c}
\frac{\mathrm{r}_{\mathrm{i}}^{2}}{2}-\frac{\mathrm{t}-\mathrm{t}_{0}}{\tau_{\mathrm{i}}}+ \\
+\frac{\varepsilon-\zeta_{\mathrm{i}}\left(\mathrm{t}_{0}\right)}{\delta_{\mathrm{i}}} \mathrm{r}_{\mathrm{i}}
\end{array}\right] \times \\
& \times\left[\Phi\left(\frac{\varepsilon-\zeta_{\mathrm{i}}(\xi)}{\delta_{\mathrm{i}}}+\mathrm{r}_{\mathrm{i}}\right)\right]_{\xi=\mathrm{t}_{0}}^{\mathrm{t}},
\end{aligned}
$$

where

$$
\begin{aligned}
& \Delta \mathrm{T}=\mathrm{T}_{1}-\mathrm{T}_{\phi}, \varepsilon=\mathrm{a}_{1}-\mathrm{x}_{0}, \\
& \xi_{\mathrm{i}}(\mathrm{t})=\mathrm{k}_{\mathrm{i}}\left[\mathrm{z}_{0} \operatorname{tg}\left(\varphi-\beta_{\mathrm{i}}\right)+\mathrm{V}\left(\mathrm{t}-\mathrm{t}_{0}\right) \sin \beta_{\mathrm{i}} \sec \left(\varphi-\beta_{\mathrm{i}}\right)\right] .
\end{aligned}
$$

Let a frame be taken at time t, i.e. the output voltages of the channels are fixed. Then $\mathrm{n}_{\mathrm{ij}}$ is a random variable, moreover $n_{i j} \in \mathrm{N}\left(0, \sigma_{i j}\right)$. We determine the parameter $\varepsilon$, characterizing the shift in the difference in radio brightness temperature relative to the projection of the MR on the xy plane, and evaluate the accuracy of its measurement in the presence of interfering parameters $T_{1}, T_{\phi}$ against the noise background $\left\{n_{i j}\right\}$.

We assume that a prior information on the parameters $\varepsilon, \mathrm{T}_{1}, \mathrm{~T}_{\phi}$ is absent.

Let us formulate the problem in terms of the estimating signal parameters theory: using the additive model (6) of the signal interaction with noise, the statistical characteristics of which are assumed to be known, it is necessary to find the optimal estimate of the unknown signal parameter $\varepsilon$ in the presence of unknown interfering parameters $T_{1}, T_{\phi}$, as well as determine the algorithm for generating the optimal estimate and its accuracy measurements [7].

Since there is no prior information on the parameters, we will use the maximum likelihood criterion as an optimal criterion, and the interfering parameters will be evaluated along with the main one. 
For the logarithm of the likelihood function of the estimated parameters $\varepsilon, \mathrm{T}_{1}, \mathrm{~T}_{\phi}$, up to an insignificant constant, we can obtain the expression.

$$
\mathrm{L}\left(\varepsilon, \mathrm{T}_{1}, \mathrm{~T}_{\phi}\right)=-\frac{1}{2} \sum_{\mathrm{i}=1}^{\mathrm{N}_{1}} \sum_{\mathrm{j}=1}^{\mathrm{N}_{2}}\left[\frac{\tilde{\mathrm{h}}_{\mathrm{ij}}-\mathrm{T}_{\phi}-\left(\mathrm{T}_{1}-\mathrm{T}_{\phi}\right) \tilde{\mathrm{f}}_{\mathrm{i}}(\varepsilon)}{\Sigma_{\mathrm{i}}}\right]^{2}
$$

The system of likelihood equations has the form:

$$
\left\{\begin{array}{l}
\left(\mathbf{h}-\mathbf{T}-\left(\mathrm{T}_{1}-\mathrm{T}_{\phi}\right) \mathbf{f}, \mathbf{f}_{\varepsilon}\right)=0 \\
\mathrm{~T}_{1}\left(\mathbf { f } \left(^{2}+\mathrm{T}_{\phi}(\mathbf{f}, \mathbf{e})=(\mathbf{f}, \mathbf{h})\right.\right. \\
\mathrm{T}_{1}(\mathbf{f}, \mathbf{e})+\mathrm{T}_{\phi}\left(\mathbf { e } \left(^{2}=(\mathbf{h}, \mathbf{e})\right.\right.
\end{array}\right.
$$

where the vector notation is introduced and the notation is used:

$$
\begin{aligned}
& \sigma_{\mathrm{i}}=\frac{\Sigma_{\mathrm{i}}}{\sqrt{\mathrm{N}_{2}}} ; \mathrm{h}_{\mathrm{i}}=\frac{1}{\sigma_{\mathrm{i}} \mathrm{N}_{2}} \sum_{\mathrm{j}=1}^{\mathrm{N}_{2}} \tilde{\mathrm{h}}_{\mathrm{ij}}, \mathrm{i} \in \overline{1, \mathrm{~N}_{1}} ; \\
& \mathbf{h}=\left(\mathrm{h}_{1}, \ldots, \mathrm{h}_{\mathrm{N}_{1}}\right) ; \mathbf{T}=\left(\mathrm{T}_{\phi} / \sigma_{1}, \ldots, \mathrm{T}_{\phi} / \sigma_{\mathrm{N}_{1}}\right) ; \\
& \mathbf{f}=\left(\tilde{\mathrm{f}}_{1} / \sigma_{1}, \ldots, \tilde{\mathrm{f}}_{\mathrm{N}_{1}} / \sigma_{\mathrm{N}_{1}}\right), \\
& \mathbf{f}_{\varepsilon}=\left(\frac{\partial \mathrm{f}_{1}}{\partial \varepsilon}, \ldots, \frac{\partial \mathrm{f}_{\mathrm{N}_{1}}}{\partial \varepsilon}\right) \text {, } \\
& \mathbf{e}=\left(\frac{1-\tilde{f}_{1}}{\sigma_{1}}, \ldots, \frac{1-\tilde{f}_{\mathrm{N}_{1}}}{\sigma_{\mathrm{N}_{1}}}\right),
\end{aligned}
$$

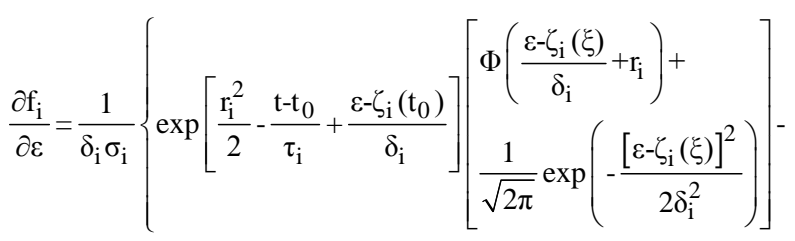

$$
\begin{aligned}
& \left.-\frac{1}{\sqrt{2 \pi}} \exp \left(-\frac{\left[\varepsilon-\zeta_{\mathrm{i}}(\mathrm{t})\right]^{2}}{2 \delta_{\mathrm{i}}^{2}}\right)\right\}_{\xi=\mathrm{t}_{0}}^{\mathrm{t}} .
\end{aligned}
$$

From the expressions (9) it can be seen that the output signals of the channels within the row must be averaged and the variance of the averaged signal is less than the $\mathrm{N}_{2}$ variance of one output channel signal.

The system of equations (8) describes an algorithm for optimal signal processing of multi-channel KENS. In the practical use of the algorithm, it is necessary to find all the solutions of the system and choose from them the one that delivers the global maximum of the likelihood function.

To do this, you must first resolve the equations linear system consisting of the last two system equations (8):

$$
\begin{aligned}
& \hat{\mathrm{T}}_{1}=\frac{\|\mathbf{e}\|^{2}(\mathbf{f}, \mathbf{h})-(\mathbf{f}, \mathbf{e})(\mathbf{h}, \mathbf{e})}{\|\mathbf{f}\|^{2}\|\mathbf{e}\|^{2}-(\mathbf{f}, \mathbf{e})^{2}}, \\
& \hat{\mathrm{T}}_{\phi}=\frac{\|\mathbf{f}\|^{2}(\mathbf{h}, \mathbf{e})-(\mathbf{f}, \mathbf{e})(\mathbf{h}, \mathbf{f})}{\|\mathbf{f}\|^{2}\|\mathbf{e}\|^{2}-(\mathbf{f}, \mathbf{e})^{2}},
\end{aligned}
$$

then substitute the values (10) in the first of equations (8) and find all its solutions using one of the known numerical methods, and then find the solution corresponding to the largest maximum of function (7).

In order to find the potential accuracy of measuring estimates, we construct the Fisher information matrix [8]:

$$
\mathbf{F}=\left[\begin{array}{ccc}
(\Delta \mathrm{T})^{2}\left\|\mathbf{f}_{\mathcal{E}}\right\|^{2} & \Delta \mathrm{T}\left(\mathbf{f}, \mathbf{f}_{\mathcal{E}}\right) & \Delta \mathrm{T}\left(\mathbf{e}, \mathbf{f}_{\mathcal{E}}\right) \\
\Delta \mathrm{T}\left(\mathbf{f}, \mathbf{f}_{\varepsilon}\right) & \|\mathbf{f}\|^{2} & (\mathbf{f}, \mathbf{e}) \\
\Delta \mathrm{T}\left(\mathbf{e}, \mathbf{f}_{\mathcal{E}}\right) & (\mathbf{f}, \mathbf{e}) & \|\mathbf{e}\|^{2}
\end{array}\right]
$$

The parameters estimates variances of $\varepsilon, T_{1}, T_{\phi}$ are determined by the diagonal elements of the inverse matrix:

$$
\begin{gathered}
\sigma_{\varepsilon}^{2}=\frac{1}{\operatorname{det} \mathbf{F}}\left[\|\mathbf{f}\|^{2}\|\mathbf{e}\|^{2}-(\mathbf{f}, \mathbf{e})^{2}\right] ; \\
\sigma_{\mathrm{T}_{1}}^{2}=\frac{(\Delta \mathrm{T})^{2}}{\operatorname{det} \mathbf{F}}\left[\|\mathbf{f}\|^{2}\|\mathbf{e}\|^{2}-\left(\mathbf{f}_{\varepsilon}, \mathbf{e}\right)^{2}\right] ; \\
\sigma_{\mathrm{T}_{\phi}}^{2}=\frac{(\Delta \mathrm{T})^{2}}{\operatorname{det} \mathrm{F}}\left[\|\mathbf{f}\|^{2}\|\mathbf{f}\|^{2}-\left(\mathrm{f}_{\varepsilon}, \mathrm{f}\right)^{2}\right],
\end{gathered}
$$

where

$$
\begin{aligned}
& \operatorname{det} \mathbf{F}=(\Delta \mathbf{T})^{2}\left\{\left\|\mathbf{f}_{\varepsilon}\right\|^{2}\left[\|\mathbf{f}\|^{2}\|\mathbf{e}\|^{2}-(\mathbf{f}, \mathbf{e})^{2}\right]-\right. \\
& -\left(\mathbf{f}, \mathbf{f}_{\varepsilon}\right)\left[\|\mathbf{e}\|^{2}\left(\mathbf{f}, \mathbf{f}_{\varepsilon}\right)-(\mathbf{f}, \mathbf{e})\left(\mathbf{e}, \mathbf{f}_{\varepsilon}\right)\right]+ \\
& \left.+\left(\mathbf{e}, \mathbf{f}_{\varepsilon}\right)\left[(\mathbf{f}, \mathbf{e})\left(\mathbf{f}, \mathbf{f}_{\varepsilon}\right)-\|\mathbf{f}\|^{2}\left(\mathbf{e}, \mathbf{f}_{\varepsilon}\right)\right]\right\}
\end{aligned}
$$

Given that $(\mathbf{f}, \mathbf{e})=\|\mathbf{f}\|\|\mathbf{e}\| \cos \|\mathbf{f}, \mathbf{e}\|$,

where $\cos \|\mathbf{f}, \mathbf{e}\|$ is the cosine of the angle between the vectors $\mathbf{f}$ and $\mathbf{e}$, formulas (11) - (13) can be simplified and the following expressions can be obtained for the standard deviations of the estimated parameters:

$$
\begin{aligned}
& \sigma_{\varepsilon}=\frac{|\sin \langle\mathbf{f}, \mathbf{e}\rangle|}{\Delta \mathrm{T}\left\|\mathbf{f}_{\mathcal{E}}\right\| \mathrm{F}\left(\mathbf{f}, \mathbf{e}, \mathbf{f}_{\mathcal{E}}\right)} ; \\
& \sigma_{\mathrm{T}_{1}}=\frac{\left|\sin \left\langle\mathbf{f}_{\mathcal{E}}, \mathbf{e}\right\rangle\right|}{\|\mathbf{f}\| \mathrm{F}\left(\mathbf{f}, \mathbf{e}, \mathbf{f}_{\mathcal{E}}\right)} ; \\
& \sigma_{\mathrm{T} \Phi}=\frac{\left|\sin \left\langle\mathbf{f}_{\mathcal{E}}, \mathbf{e}\right\rangle\right|}{\|\mathbf{e}\| \mathrm{F}\left(\mathbf{f}, \mathbf{e}, \mathbf{f}_{\mathcal{E}}\right)}
\end{aligned}
$$


Where

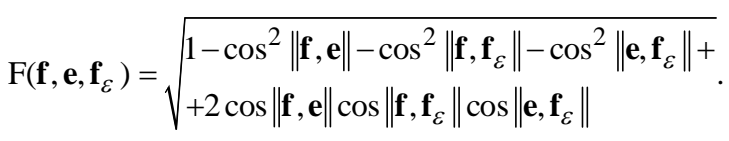

Relations (14) are generalized and can be simplified in case that the sensitivity of all the radiometer channels is the same and equal to $\sigma$.

\subsection{The results of a quantitative assessment of the KENS accuracy radiometric characteristics}

An expressions analysis (14) shows that the parameter estimation accuracy $T_{1}, T_{\phi}$ does not depend on the values of these parameters. The accuracy of the KENS positioning is inversely proportional to the ratio $\Delta \mathrm{T} / \sigma$, which is the signal-to-noise ratio for the simplest image in the form of a temperature difference along a straight orthogonal sight plane.

The accuracy positioning assessment is feasible based on the construction of dependencies $\sigma_{\varepsilon}(\varepsilon), \quad \sigma_{\mathrm{T}_{1}}(\varepsilon)$, $\sigma_{\mathrm{T}_{\phi}}(\varepsilon)$ according to formulas (14). To do this, we will accept the following MR model flight conditions and SS sighting:

- the height of the MR above the $\mathrm{H}_{0}=0,5 \mathrm{~km}$;

- flight speed MR V=0,5 km/s;

- number of rows $\mathrm{N}_{1}=8$;

- angle of sight $\varphi=20^{\circ}$;

- the APP aperture angles at half power in the elevation plane $\theta_{\mathrm{x}}$ and $\theta_{\mathrm{y}}$ in the azimuth plane are $\theta_{\mathrm{x}}=\theta_{\mathrm{y}}=2^{\mathrm{o}}$;

- reduced sensitivity $\sigma_{\mathrm{r}}=\mathrm{K}_{\mathrm{r}} \mathrm{T}_{\mathrm{n}} / \sqrt{\Delta \mathrm{f}}=0.5 \frac{\text { degree }}{\mathrm{s}}$;

$-\mathrm{N}_{2}=1 ; \mathrm{x}_{0}=\mathrm{y}_{0}=0$.

To plot the desired dependencies, we first determine the time constant to the radiometric channel t. To do this, in condition that, during the frame time exposure, the distance by which the section center of the partial APP is shifted does not exceed the section half-width of the main ellipse semi-axis $\Delta \mathrm{x}_{\mathrm{ij}}(\mathrm{t})$. In a plane that passes through points: $\left(\mathrm{x}_{0}, 0, \mathrm{z}_{0}\right),\left(\mathrm{x}_{0}, 0,0\right),\left(\mathrm{x}_{\mathrm{ij}}, \mathrm{y}_{\mathrm{ij}}, 0\right)$ value $\Delta \mathrm{x}_{\mathrm{ij}}(\mathrm{t})$ determined by the formula [8]:

$$
\Delta x_{i j}(t)=z(t)\left[k_{i j}(t)-1\right] \operatorname{ctg}\left(\theta_{x_{i j}} / 2\right)
$$

For these conditions, the time constant of the radiometric channel $\tau$ will be equal to:

$$
\tau=\frac{\Delta_{\mathrm{x} 1} \cos \left(\varphi-\beta_{1}\right)}{3 \mathrm{~V} \sin \beta_{1}}=0,049 \mathrm{c} .
$$

Accordingly, the radiometric channel sensitivity will be:

$$
\sigma=\sigma_{\text {пр }} \tau^{-1 / 2}=2,259 \mathrm{~K}
$$

$\mathrm{P}$ The results of a numerical assessment of the accuracy characteristics are given in the form of a graph in Figure 3. During calculations, the signal-to-noise ratio in channels was used $q=\Delta T / \sigma=3$.

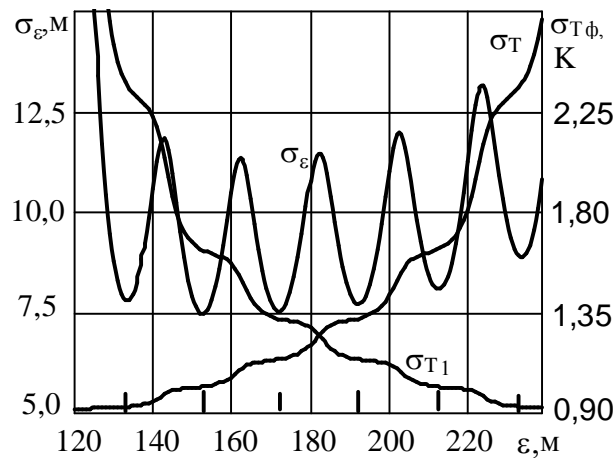

Figure 3 : Dependency $\sigma_{\varepsilon}(\varepsilon), \quad \sigma_{\mathrm{T}_{1}}(\varepsilon), \quad \sigma_{\mathrm{T}_{\phi}}(\varepsilon)$ graphs

The dependence $\sigma_{\varepsilon}(\varepsilon)$ analysis shows that the measuring $\varepsilon$ accuracy parameter $\mathrm{e}$ is the best for temperature difference $\varepsilon$, positions, which coincide with one of the partial APP axes, and is the worst in cases where the position of the temperature difference is located exactly in the middle between the axes of the neighboring APPs. The higher the temperature measuring accuracy $T_{1}, T_{\phi}$ of the boundary regions, the greater the number of the antenna rays system covers the corresponding region.

For images where the anchor object occupies a significant part of the sensor's view field, a significant improvement in the MR navigation is possible compared to the resolution element of the antenna system on the ground. So, for example, with an average distance between the axes of the APPs adjacent sections of the $20 \mathrm{~m}$ order for the signal-to-noise ratio $\mathrm{q}=5 \ldots 9$ the accuracy of positioning will be $\sigma_{\varepsilon}=(4,5 \ldots 2,5) \mathrm{M}$.

\section{CONCLUSION}

A model is proposed to describe the SS when navigating low-altitude MR equipped with KENS in the form of a step difference in the informative parameter, which is well described by the generalized telegraph process and takes into account the SS correlation properties. The model feature is its application in a wide spectral range, regardless of the informative parameter type.

The KENS potential accuracy assessing relations for the step type of image models are obtained .A numeric assessment of the KENS potential accuracy positioning for typical model conditions for the MR use with the SS images in the form of a generalized telegraph 
process was carried out. Estimates for the informative parameter used in radiometric navigation systems, which can be similarly obtained for other types of informative parameters are made.

The achieving possibility of the KENS accuracy positioning of the several units meters order is shown.

\section{REFERENCES}

1. V.K. Baklytskyu. Signal methods in extreme correlation navigation systems, Radio and communication, Moskov, USSR, 1986, $216 \mathrm{p}$.

2. N. Yeromina, A. Sotnikov, V. Tarshyn. Form of the virtual functions of the correlation-extremal systems of navigation for the criterion of the outraged correlation of the correlation of the correlation of the correlation, News of NTU "KhPI", Ukraine, Kharkiv, 2016, №50(1222), pp. 68-73.

3. A. Sotnikov, V. Tarshyn, N. Yeromina, S. Petrov, N. Antonenko. A method for localizing a reference object in a current image with several bright objects, Eastern-European Journal of Enterprise Technologies. 2017. Vol. 3. № 9 (87). pp. 68-74.

4. A.M. Sotnikov. Models of streamlined images that are formed by channels of a combined correlation-extremal system of a navigable pilotless vehicle, Advanced information technology in the field of security and defense, Ukraine, Kyiv, 2018, pp. 29-38.
5. A.M. Sotnikov. Preparation of reference images for high-speed correlation-extreme navigation systems based on the use of direct correlation analysis, Science and Technology of the Reinforced Forces of the Ukrainian Forces of Ukraine,Ukraine, Kharkiv, 2015, pp. $69-73$.

6. O. Vorobiov, A. Sotnicov, A Tantsiura. Models of current images that are formed by the combined correlation-extremal navigation system of a non-flammable aircraft. Modern information technologies in the sphere of security and defence. Ukraine, Kyiv, — 2018. Vol.2, No.32. - pp. 29-36.

7. N.Yeromina, S. Petrov, A. Tantsiura, M. Iasechko, V. Larin. Formation of reference images and decision function in radiometric correlation-extremal navigation systems. Eastern-European Journal of Enterprise Technologies. - 2018. Vol.4, No.9 (94). — pp. 27-35. DOI: 10.15587/1729-4061.2018.139723

8. E. Kulikov, A. Triphonov. Estimation of signal parameters against a background of interference. Soviet radio, Moskov, USSR, $1978,296 \mathrm{p}$.

9. A. Tantsiura, A. Bondarchuk, O. Ilin, Yu. Melnyk, O. Tkachenko, K. Storchak The image models of combined correlation-extreme navigation system of flying robots. International Journal of Advanced Trends in Computer Science and Engineering. 8(4), JulyAugust 2019, pp. $1012 \quad$ - 1019 DOI: 10.30534/ijatcse/2019/05842019 